\title{
COST OF TRANSMISSION TRANSACTIONS: Comparison and Discussion of Used Methods
}

\author{
Judite Ferreira ${ }^{1}$, Zita Vale ${ }^{2}$, A. Almeida Vale ${ }^{3}$ and Ricardo Puga ${ }^{4}$ \\ ${ }^{1,2,4}$ Polytechnic Institute of Porto (IPP)/Institute of Engineering (ISEP)/Department of Electrical Engineering (DEE) \\ Rua Dr. António Bernardino de Almeida - 4200 - 072 - Porto- Portugal \\ phone: 351-22-8340500, fax: 351-22-8321159, \\ e-mail: mjudite@dee.isep.ipp.pt, zav@dee.isep.ipp.pt, rpuga@dee.isep.ipp.pt
}

${ }^{3}$ University of Porto (UP)/Scholl of Engineering (FEUP)/Department of Electrical Engineering and Computers (DEEC) phone: 351-22-5081861 fax: 351-22-5081443

e-mail: aav@,fe.up.pt

\begin{abstract}
Power market deregulation imposed the separation of several activities such as power production, transmission and distribution, with the consequent separation of these activities costs.

This article presents and compares methods that allow determining how much and who must pay for the use of the electrical transmission network. This issue is analysed, under the point of view of several transmission system users: generators, loads and transactions being analysed. The advantages and disadvantages of each method, from the point of view of the considered users are discussed.

The embedded methods are used as the base of costs calculation, specifically the methods Postage-Stamp and MW-mile and some of its variants. This study is illustrated using a simple nine-bus electrical network that being used by R\&D groups for several projects concerning power markets.
\end{abstract}

\section{Keywords}

Power transmission economics, Power transmission lines, Wheeling transaction, Transmission pricing, Embedded methodology.

\section{Introduction}

In the competitive electric energy market it is important to quantify efficiently the costs associated to each activity in the sector $[1,2]$.

The main costs associated to the market of electric energy are the costs of production, costs of transmission and costs of distribution. This paper studies and calculates the transmission costs, associated to a wheeling transaction.

The study case presents a set of twenty transactions that correspond to bilateral contracts between generators and loads. The electric network used in this study is represented in the Fig. 1.

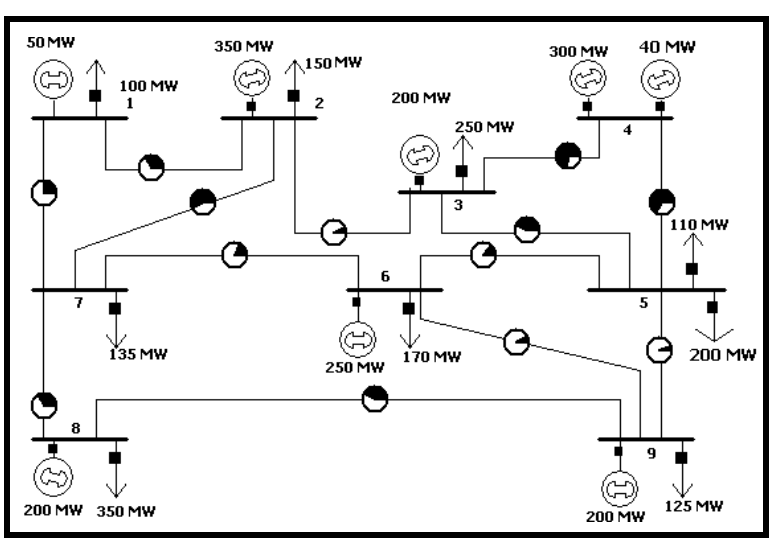

Fig. 1: Example Network

For calculation of the taxes to be impute to each $\mathrm{R}(\mathrm{u})$ transaction the embedded methodology was used. The development tools used are: Matlab for implementation of the embedded methods and the Power World simulator to carry out the simulation of Power Flow.

\section{Theoretical Development}

In the embedded methods all system costs, "existing transmission system", "operating" and "expansion", these costs are allocated among system user in proportion to their "extent of use" of the transmission resources.

Allocation methodologies differ on their definition and measure of this "extent of use". They can be classified as load flow based methods and rolled-in methods such MW-mile and Postage-Stamp methodology respectively [3]. The main values difference between the Postage-Stamp and MW-mile methods is that: the MW-mile uses the calculated values of power flows in its implementation, while the Postage-Stamp method does not use this calculation. The studied methods are: Methods of Rolled type in Postage-Stamp, MW-mile 
(classic), Base, Module or Use, Zero Counterflow and Dominant Flow.

To the long one of the related analysis, the variable and expressions are used to follow presented:

$k$ - Circuit that binds bus $i$ with bus $j$

$C_{k}$ - Cost of line $k$, (kEuro)

$F M_{k}$ - Line $k$ capacity (MW)

$F_{k}$ - Flow in line $k$ in the initial conditions (MW)

$L_{k}$ - Length of the line $k(\mathrm{~km})$

$C T=\sum_{k} C_{k}-$ Total cost of transmission (kEuro)

$F_{k}(u) \quad$ - Impact of transaction $u$ in line $k(\mathrm{MW})$

$W(u) \quad$ - Power of transaction $u$ (MW)

$R(u) \quad$ - Allocated cost to agent $u$ (kEuro)

$P G(g)$ - Power produced for the generator $g$

\section{A - Methods of Rolled type in Postage-Stamp}

This method is of simple implementation. Its calculation is based on the amplitude of the power transaction. The measure of this amplitude normally is gotten at the moment where the system reaches maximum power. Being the tariff, represented by $\mathrm{P}$, the same for all the transactions and $\mathrm{R}(\mathrm{u})$ the tax to assign to each transaction $\mathrm{u}$ proportional to $\mathrm{P}$. The expression 2 allows the calculating of R(u) [3].

$$
\begin{aligned}
& P=\frac{C T}{\sum_{g} P G(g)} \\
& R(u)=P \times W(u)
\end{aligned}
$$

This method presents some disadvantages: it does not consider the actual state of the system so it because of this does not give true economic information to the users. Does not induce a load increase in the zones where exists bigger production, neither the installation of new generators in areas with bigger consumption. For example independent generators do have not incentives. With this method a transactions in which the load and the generator are close, what means using lightly the system, may have to subsidize other transactions that use much more the system. Moreover, this method does not consider the network and the power flows involved. The methodologies also presented in sections $B, C, D, E, F$, are based on the calculations of power flow with intention to consider the state of the network.

\section{$B$ - MW-Mile (classic)}

This methodology is of simple implementation. It considers the contributions of each transaction to the power flows in the lines and the amount used of network for each transaction. The expression (4) shows how to determine the tax to be paid for the $\mathrm{R}(\mathrm{u})$ transaction [3].

$$
\begin{gathered}
P=\frac{C T}{\sum_{k} F_{k} \times L_{k}} \\
R(u)=\sum_{k} P \times F_{k}(u) \times L_{k}
\end{gathered}
$$

However, this method has some disadvantages. In fact, it is only applicable to bilateral transactions where the points of injection and reception are known. For that reason its use is impracticable in a Pool. This method considers that all the negative flows associated to a transaction are beneficial to the network. However, this only has real means in the case of the lines near the limits. Another inconvenience of this method is that it does not have an economic basis. Therefore it does not transmit adequate economic signals to the users, for example indicating is the availability of the equipment.

The methods presented in next sections reduce the disadvantages of the MW-mile (classic).

\section{C-Base}

There is a great difference between this method and the original MW-mile [4]. While the original method considers the maximum capacity of the line in denominator, this method uses the total flow of the line. With this method the total cost of the system is distributed by all the transactions, according to the expression (5) that allows to calculate $\mathrm{R}(\mathrm{u})$.

$$
R(u)=\sum_{k} C_{k} \frac{F_{k}(u)}{\sum_{s} F_{k}(s)}
$$

With this method, some of the R(u) taxes can be negative, what it means that one definitive transaction can receive a credit to use the transmission system. This is always verified when active power flow provoked by this transaction is in contrary direction to the one of the active power flow in the initial conditions of the system. Effectively, this fact alone is important in the case of the line work near to its capacity, in case that if it does not verify, some of the users of the network pay sufficiently, in benefit of that receives but that in the reality they had not brought benefit some for the operation of the system.

\section{D - Module or Use}

This method distributes the total cost of the system for the different transactions, considering transactions in both directions. In this way, all pay, but the cost is more distributed, becoming cheaper for the cases where the transaction does not reduce the flows in the lines. The expression (6) shows how to determine the tax to be paid for the $\mathrm{R}(\mathrm{u})$ transaction [3]. 


$$
R(u)=\sum_{k} C_{k} \frac{\left|F_{k}(u)\right|}{\sum_{s}\left|F_{k}(s)\right|}
$$

The difference of this method in relation to the MW-mile (Base) is that it considers the absolute values of the line flows originated by transaction $\mathrm{u}$, instead of his signed values.

\section{E- Zero Counterflow}

The Zero Counterflow method only taxes the positive flows. This method assumes that the negative flows are beneficial for the network, therefore in these cases the transactions are not paid but also they do not have credit. The expression (7) shows how to determine the tax to be paid for the $\mathrm{R}(\mathrm{u})$ transaction [3].

$$
R(u)= \begin{cases}\sum_{k} C_{k} \frac{F_{k}(u)}{\sum_{s} F D_{k}(s)} & \text { for } F_{k}(u)>0 \\ 0 & \text { for } F_{k}(u) \leq 0\end{cases}
$$

$$
F D_{k}(u)=\left\{\begin{array}{cc}
F_{k}(u) & \text { if } F_{k}(u)>0 \\
0 & \text { if } F_{k}(u) \leq 0
\end{array}\right.
$$

The function $F D_{k}(u)$ only considers the impact, provoked for the transaction $u$ in line $k$, when these increase the active power flow in this line.

\section{F - Dominant Flow}

This method is the join of the two previous methods. It is considered that $\mathrm{R}(\mathrm{u})$ is the addition of two parcels $\mathrm{RA}(\mathrm{u})$ and $\mathrm{RB}(\mathrm{u})$. The parcel $\mathrm{RA}(\mathrm{u})$ is determined using the Zero Counterflow method substituting cost $\mathrm{C}_{\mathrm{k}}$ for $\mathrm{CA}_{\mathrm{k}}$. The parcel $\mathrm{RB}(\mathrm{u})$ is determined using the "Modulo or Use" method where $\mathrm{C}_{\mathrm{k}}$ is substituted by $\mathrm{CB}_{\mathrm{k}}$. Factor $\mathrm{CA}_{\mathrm{k}}$ corresponds to the cost due to the transit in the line for the base case of the system and $\mathrm{CB}_{\mathrm{k}}$ corresponds to the cost of the not used capacity. The expression (9) shows how to determine the tax to be paid for the $\mathrm{R}(\mathrm{u})$ transaction [3].

$$
R(u)=R A(u)+R B(u)
$$

$$
\begin{aligned}
& \left\{A(u)= \begin{cases}\sum_{k} C A_{k} \frac{F_{k}(u)}{\sum_{s} F D_{k}(s)} & \text { For } F_{k}(u)>0 \\
0 & \text { For } F_{k}(u) \leq 0\end{cases} \right. \\
& R B(u)=\sum_{k} C B_{k} \frac{\left|F_{k}(u)\right|}{\sum_{s}\left|F_{k}(s)\right|} \\
& \left\{\begin{array}{l}
C A_{k}=C_{k} \frac{F M_{k}-F_{k}(u)}{F M_{k}} \\
C B_{k}=C_{k} \frac{F_{k}(u)}{F M_{k}}
\end{array}\right.
\end{aligned}
$$

With this method all the participants that use the system in the opposite direction of the resultant flow receive an incentive, which consists of lesser cost. This incentive increases when the system is more loaded, arriving to zero cost when the system is to the maximum load. These economical signs are coherent with the intents of reducing expansion costs.

\section{Study Case}

For this study case are calculated the imputed taxes for the 20 transactions that are presented in the scenario presented in table I and table II. These transactions are relative to the bilateral contracts carried through between generators and loads that integrate the network presented in figure 1 .

\begin{tabular}{|c|c|c|c|c|c|c|}
\hline & Load & L1 & L2 & L3 & L51 & L52 \\
\hline Ger & (MW) & 100 & 150 & 250 & 110 & 200 \\
\hline G1 & 50 & 0 & $\mathrm{~T} 2-50$ & 0 & 0 & 0 \\
\hline G2 & 350 & T1-100 & 0 & 0 & 0 & 0 \\
\hline G3 & 200 & 0 & T3-100 & 0 & 0 & 0 \\
\hline G41 & 300 & 0 & 0 & 0 & T5-100 & T7-160 \\
\hline G42 & 40 & 0 & 0 & 0 & T6-10 & 0 \\
\hline G6 & 250 & 0 & 0 & $\mathrm{~T} 4-250$ & 0 & 0 \\
\hline G8 & 200 & 0 & 0 & 0 & 0 & T8-40 \\
\hline G9 & 200 & 0 & 0 & 0 & 0 & 0 \\
\hline
\end{tabular}

In this study case all the produced power is commercialised by bilateral contracts for the period of one year. It is considered the pair generation/load, as are respectively the production and its associated load to one determined wheeling agent, or traditional utility.

Table I: Scenarios of Transactions (MW)

Where: 
Table II: Scenarios of Transactions (continuation)

\begin{tabular}{|c|c|c|c|c|c|}
\hline & Load & L6 & L7 & L8 & L9 \\
\hline Ger & (MW) & 170 & 135 & 350 & 125 \\
\hline G1 & 50 & 0 & 0 & 0 & 0 \\
\hline G2 & 350 & T9-30 & T12-20 & T15-100 & T18-100 \\
\hline G3 & 200 & 0 & T13-100 & 0 & 0 \\
\hline G41 & 300 & T10-40 & 0 & 0 & 0 \\
\hline G42 & 40 & 0 & T14-15 & 0 & T19-15 \\
\hline G6 & 250 & 0 & 0 & 0 & 0 \\
\hline G8 & 200 & T11-100 & 0 & T16-50 & T20-10 \\
\hline G9 & 200 & 0 & 0 & T17-200 & 0 \\
\hline
\end{tabular}

The simulator used to obtain the power flow in the transmission lines was the "Power World" Simulator. First, was been determinate the power flow at the base state of the system, presented on $\mathrm{F}_{\mathrm{k}}$, with all the loads and generators connected in the network and with not resistance lines consideration. To calculate the contribution due to each transaction $u$ (pair generator/load), is removed the pair associated to it and in this situation the power flow is carried out with a new simulation. Analysing the results of these two simulations allow to calculate the power flows in the $\mathrm{F}_{\mathrm{k}}(\mathrm{u})$ lines, associates to the transaction $\mathrm{u}$. This process is repeated for all the transactions.

Using the embedded methods, and knowing the contributions of each transactions in each line, is calculated $\mathrm{R}(\mathrm{u})$ the tax to assign to each transaction $\mathrm{u}$. The Matlab was the programming tool used to the implementation of the exposed methods. The software architecture is presented in Fig. 2.

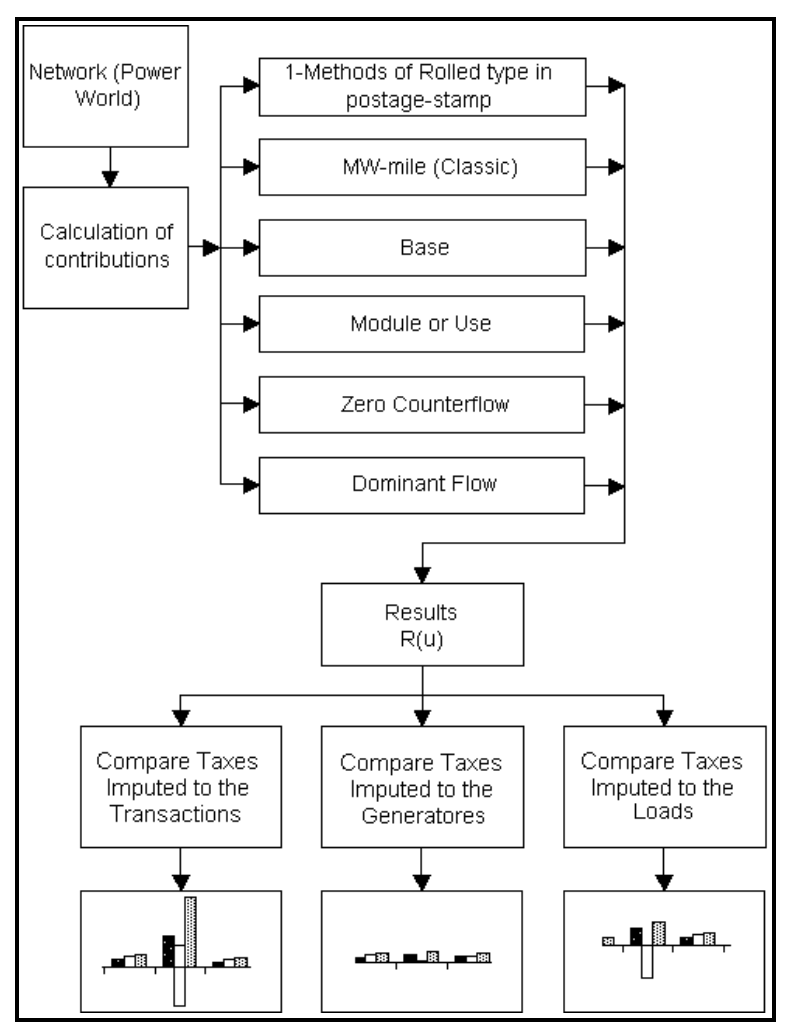

Fig. 2: Software Architecture

\section{Results}

After the accomplishment of all the simulations that had allowed calculating the taxes to impute to the 20 transactions had been gotten the results presented in table VIII of the appendix A. With these results comparisons are carried through that allow getting some conclusions presented in section 5 .

The total cost of transmission is $65707 \mathrm{kEuro}(\mathrm{CT})$. It is calculated appealing of attributed cost $C_{k}$ to each line (values presented in table VII of the appendix A), according to following expression:

$$
C T=\sum_{\text {all lines } k} C_{k}
$$

$\mathrm{CT}=\mathrm{C}_{1-2}+\mathrm{C}_{1-7}+\mathrm{C}_{2-3}+\mathrm{C}_{2-7}+\mathrm{C}_{3-4}+\mathrm{C}_{3-5}+\mathrm{C}_{4-5}+\mathrm{C}_{5-6}+$ $\mathrm{C}_{5-9}+\mathrm{C}_{6-7}+\mathrm{C}_{6-9}+\mathrm{C}_{7-8}+\mathrm{C}_{8-9}$

$\mathrm{CT}=65707 \mathrm{kEuro}$.

The Taxes imputed to each transaction are presented in the table VIII of the appendix A. With these values, it is possible to compare the imputed cost to some of the intervening ones in the studied scenario. These costs are compared to the transactions, generators and loads that present similar characteristics, such as: transactions, generators value and loads with similar value.

\section{A - Compare Taxes Imputed to the Transactions}

\section{Transactions of $100 \mathrm{MW}$}

The transactions $\mathrm{T} 1$ and $\mathrm{T} 15$ have the same transaction power $(100 \mathrm{MW})$, however are taxed with different values as presented in the graph of Fig. 3 and in the table III.

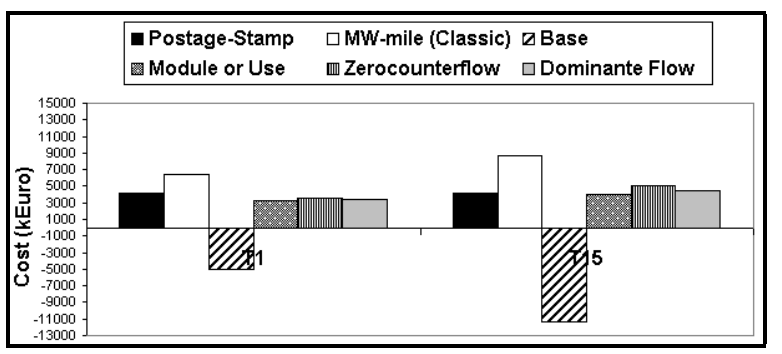

Fig. 3: Taxes Imputed to the Transactions T1 and T15

The two transactions pay different values, because the distance from loads to generators, that correspond to each one of the transactions also are different. The fact of the value taxed to T15 is bigger than the other transaction is due to the fact of these uses more the network and is more remote from the production contract point. 
Table III: Taxes Imputed to the Transactions T1 and $\mathrm{T} 15$

\begin{tabular}{|c|c|c|}
\cline { 2 - 3 } \multicolumn{1}{c|}{} & T1 & T15 \\
\hline Postage-Stamp & 4133 & 4133 \\
\hline MW-mile (Classic) & 6405 & 8668 \\
\hline Base & -4966 & -11341 \\
\hline Module or Use & 3233 & 3971 \\
\hline Zerocounterflow & 3566 & 4997 \\
\hline Dominante Flow & 3474 & 4440 \\
\hline \hline
\end{tabular}

\section{Transactions of $50 \mathrm{MW}$}

Considering the T2 and T16 transactions, worthy like $50 \mathrm{MW}$, the values to impute are presented in the table IV and represented graphically in the figure 4.

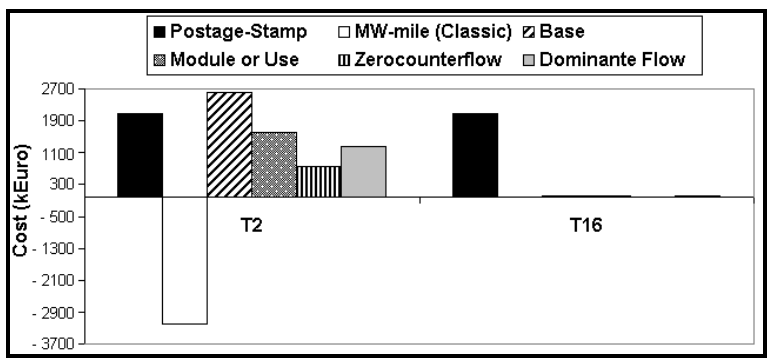

Fig. 4: Taxes Imputed to the Transactions T2 and T16

The T16 transaction is taxed in almost all the methods with practically null values, compared with the total cost of transmission. The exception is the Post Stamp method where the transaction is taxed in 3,14\% (2066 kEuro). This happens because the load L8 and the generator G8 are located in the same bus and they are the implicated in the transaction T16.

Table IV: Taxes Imputed to the Transactions T2 and T16

\begin{tabular}{|c|c|c|}
\cline { 2 - 3 } \multicolumn{1}{c|}{} & T2 & T16 \\
\hline Postage-Stamp & 2066 & 2066 \\
\hline MW-mile (Classic) & -3186 & -9 \\
\hline Base & 2597 & 5 \\
\hline Module or Use & 1623 & 5 \\
\hline Zerocounterflow & 758 & 1 \\
\hline Dominante Flow & 1256 & 2 \\
\hline
\end{tabular}

The values in the table IV, for the taxes imputed to the transaction T16, are not exactly zero, what is justified due to the rounding of the calculations.

By the analysis of the graph represented in figure 4 , it is verified that $\mathrm{T} 2$ is taxed with positive value in the methods Post-Stamp, Base, Module or Use, Zero Counterflow and Dominant Flow and with negative value for the method MW-mile Classic.
The difference of the value of the taxes imputed to the transactions $\mathrm{T} 2$ and $\mathrm{T} 16$, that have the same contractual value of power, it is because the contract between the load L2 and the generator G1 (that give origin to the T2 transaction) are not connected in the same bus.

\section{$B$ - Compare Taxes Imputed to the Generators}

Analysing from the point of view of the generators, for example generators producing $200 \mathrm{MW}$, the tax to impute is represented in the graphic of the Fig. 5 and table V.

In this analysis it is verified that the methods where the taxed values are more identical are the Module or Use, Zero Counterflow and Dominant Flow. Although this methods don't give credit to the generators but they tax them with less values. For example the generator G8 in these last three methods does not receive, but is what pay less. With this analysis was concluded that the generators that inject the same power could be taxed with different values. These values depends on with and who the producers have its contracts, and which the effect of these contracts in the actives powers flows.

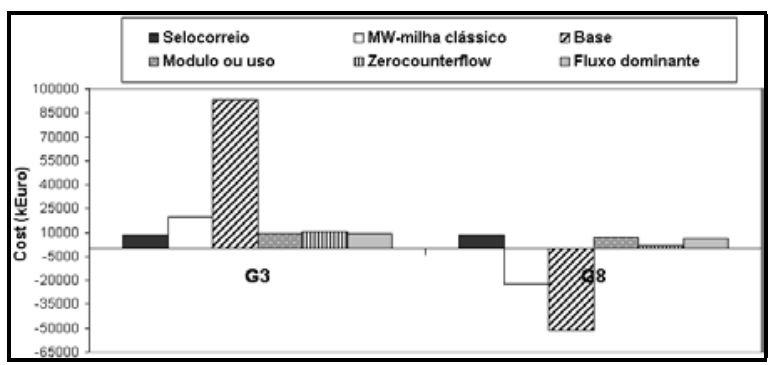

Fig. 5: Taxes Imputed to the Generators G3 and G8

Analysing the graphic of Fig. 4, we can conclude that G3 regarding the G8 pays always the same or more, being G8 be able to receive. This happen, when the methods MW-mile classic and Base are used.

Table V: Taxes Imputed to the Generators G3 and G8

\begin{tabular}{|c|c|c|}
\cline { 2 - 3 } \multicolumn{1}{c|}{} & G3 & G8 \\
\hline Postage-Stamp & 8265 & 8265 \\
\hline MW-mile (Classic) & 19649 & -22654 \\
\hline Base & 93299 & -51512 \\
\hline Module or Use & 9075 & 6822 \\
\hline Zerocounterflow & 10584 & 2015 \\
\hline Dominante Flow & 9231 & 5781 \\
\hline
\end{tabular}

The generator G8 has greater number of transactions regarding the G3, for the same total power transacted. Therefore, we can conclude, that may be more advantageous to the generator make several transactions, when the network is used. 


\section{C - Compare Taxes Imputed to the Loads}

The aim of the comparisons, of the taxes imputed to the loads, is verify which the approach are more economical advantageous, if the utilization of the transmission network is pay by consumers.

The values presented in table VIII, are gotten adding the taxes imputed to all the transactions associated with the contracts makes for one load. For example, the value to tax to the load L5_1, was gotten adding the taxed values of the transactions $\mathrm{T} 5$ and $\mathrm{T} 6$ that are associates to the load L5_1. The values of these taxes are presented in table VI and figure 6.

\section{Compare Taxes Imputed to the Loads L1 and L5 1}

The loads L1 and L5 1 have slightly different values of active power, this is the load L5_1 has a value of active power $10 \%$ greater that the L1 load. These loads have the following acts of contract:

\section{- The L1 load contracts $100 \mathrm{MW}$ to the G2 generator.}

- The L5_1 load contracts to the generators G4_1 the powers of $100 \mathrm{MW}$ and $10 \mathrm{MW}$ to the G4 2 .

This contracts are carried through in two cases between consecutive buses, however the line length that connect bus 1 to bus 2 are of $405 \mathrm{~km}$ and the distance between bus 4 and bus 5 are of $578 \mathrm{~km}$, what means that the L5_1 load is about $173 \mathrm{~km}$ more distant from the power injection point than the L1 load.

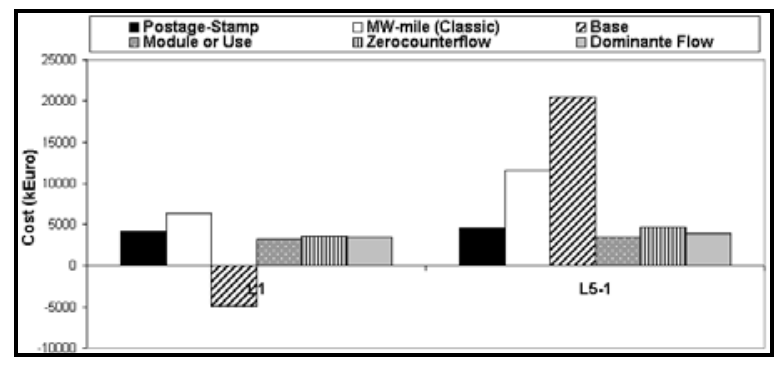

Fig. 6: Taxes Imputed to the Loads L1 and L5_1

Analysing the values in the table VI and observing the graph of figure 6 , it is verified that for all the six studied methods the load L5_1 pay always more than the $\mathrm{L} 1$ load. The values of the taxes that are in the column identified for $(\mathrm{L} 1+10 \% \mathrm{~L} 1)$, correspond to a auxiliary calculation that determines the value of the taxes imputed to L1 increased in $10 \%$.
Table VI: Taxes Imputed to the Loads L1 and L5_1

\begin{tabular}{|c|c|c|c|}
\cline { 2 - 4 } \multicolumn{1}{c|}{} & L1 & L1+10\%L1 & L5_1 \\
\hline Postage-Stamp & 4133 & 4546 & 4546 \\
\hline MW-mile (Classic) & 6405 & 7046 & 11596 \\
\hline Base & -4966 & -5463 & 20545 \\
\hline Module or Use & 3233 & 3556 & 3396 \\
\hline Zerocounterflow & 3566 & 3922 & 4665 \\
\hline Dominante Flow & 3474 & 3821 & 3967 \\
\hline
\end{tabular}

The taxes imputed to the L1 load vary between, a value where the load receives 4966 kEuro $(7,56 \%$ absolute value), calculated by the method Base and 6405 a maximum value to pay of kEuro $(9,75 \%$ of the total cost) taxed by the Classic MW-milha method. In the case of the L5_1 load the boundary-values are of a minimum to pay of $3396 \mathrm{kEuro}(5,17 \%)$ calculated by the method Module or Use and a maximum of 20545 kEuro $(31,27 \%)$ to pay calculated by the method Base. It is also verified that the taxes calculated by the method Postage-Stamp had increased proportionally, with the increase of the value of the power contracted for the load. For the remaining methods the values taxed to L5_1 increase more than $10 \%$ of the value taxed to L1, as can be analysed in table VI.

For the comment of the graph of figure 6 it is a fact, that the price to pay for the loads to the concessionaire of the network is very variable, being the three last methods Module or Use, Zero Counterflow and Dominant Flow, the most advantageous for the load, although the load haven't the possibility to receive, such happens in the method MW-mile classic in the case of the load L1.

\section{Conclusion}

All the taxes calculated for the methods presented in this work pay the total cost of transmission, as it can be verify in table VIII of the appendix A.

The method Postage-Stamp taxes all the transactions in function of the contracted power value and the total transmission cost. What it implies for one electrical system, that all the transactions with the same value of power pay the same for the use of the transmission network, just that they do not use it. For example, a transaction between producers and consumers on the same bus, that don't need use the electrical lines for the physical concretisation of the transaction, is taxed in the same way that another one, that carries through between producers and consumers who are in different bus, needing therefore to use the network.

In relation to the method Base, we conclude that this method allow that the transactions receive for the use of the network, although when they are taxed positively pays very high values. We can be conclude that this method isn't advantageous to the transactions, because it provokes great difference in the values taxed to the transactions with equal power transacted. 
The taxes calculated for the method MW-mile classic present the same tendency that the values taxed by the method Base, however with lesser amplitude of the taxed values.

For the methods, Module or Use, Zero Counterflow and the Dominant Flow, we conclude that they always tax the transactions with a positive value. Although this values are smaller and more uniform, comparing identical transactions. These taxes in certain situations give some incentives, but they do not provoke therefore a great increase of taxes for the transactions where this benefit if does not verify.

The methods, MW-mile classic, Base, Module or Use, Zero Counterflow and the Dominant Flow, tax in function of the impacts in the actives powers flows or in function of the distance from the load point and the injection point. This result in a null value for the taxes in the cases where the load and the generator are in the same bus and the power injected for the generator is equal or superior to the one of loads.

\section{References}

[1] Isabel Praça, Carlos Ramos, Zita Vale, Manuel Cordeiro, "A Multi-Agent Simulation Prototype Decision Support in Electricity Market”, 2002 AI, Simulation and Planning in High Autonomy Systems, Lisbon Portugal, pp. 247-252, April 2002.

[2] Isabel Praça, Carlos Ramos, Zita Vale, Manuel Cordeiro, "A Multi-Negotiation Period Prototype for Competitive Electricity Markets Simulation", International Conference on Fuzzy Systems and Soft Computational Intelligence in management and Industrial Engineering, Istambul, Turquia, pp. 84-91, May 2002.

[3] D Shirmohammadi, C Rajagopalan, E R Alward, C L Thomas, " COST OF TRANSMISSION TRANSACTIONS: AN INTRODUCTION”, Transactions on Power Systems, vol. 6, No. 4, November 1991.

[4] D Shirmohammadi, X V Filho and B Gorenstin, M V P Pereira, " SO ME FUNDAMENTAL TECHINICAL CONCEPTS ABOUT COST BASED TRASMISSION PRICE " IEEE Transactions on Power Systems, vol. 11, No. 2, November 1996.

\section{Appendix A}

Table VII: Electrical Characteristics Lines

\begin{tabular}{|c|c|c|c|c|c|c|}
\hline Line & $\mathbf{R}$ & $\mathbf{X}$ & $\mathbf{C}$ & $\mathbf{L}$ & Lim & Ck \\
\hline & (p.u.) & (p.u.) & (p.u.) & (Km) & (MW) & (kEuro/yr) \\
\hline 1_2 & 0,070 & 0,200 & 0,020 & 578 & 300 & 7340 \\
\hline 1_7 & 0,050 & 0,200 & 0,020 & 289 & 200 & 3670 \\
\hline 2_3 & 0,080 & 0,300 & 0,030 & 463 & 200 & 5880 \\
\hline 2_7 & 0,050 & 0,250 & 0,030 & 289 & 200 & 3670 \\
\hline 3_4 & 0,050 & 0,100 & 0,010 & 289 & 200 & 3670 \\
\hline 3_5 & 0,060 & 0,300 & 0,020 & 578 & 200 & 7340 \\
\hline 4_5 & 0,030 & 0,200 & 0,025 & 405 & 300 & 5900 \\
\hline 5_6 & 0,120 & 0,260 & 0,025 & 694 & 200 & 7813 \\
\hline 5_9 & 0,020 & 0,100 & 0,010 & 116 & 200 & 1473 \\
\hline 6_7 & 0,200 & 0,400 & 0,040 & 1156 & 200 & 6468 \\
\hline 6_9 & 0,100 & 0,300 & 0,030 & 578 & 200 & 7340 \\
\hline 7_8 & 0,020 & 0,100 & 0,010 & 116 & 200 & 1473 \\
\hline 8_9 & 0,050 & 0,200 & 0,020 & 289 & 200 & 3670 \\
\hline
\end{tabular}

Table VIII: Taxes Imputed to the Transaction

\begin{tabular}{|c|c|c|c|c|c|c|}
\hline T & Post & Class & Base & Mod & Count & Domin \\
\hline $\mathbf{1}$ & 4133 & 6405 & -4966 & 3233 & 3566 & 3474 \\
\hline $\mathbf{2}$ & 2066 & -3186 & 2597 & 1623 & 758 & 1256 \\
\hline $\mathbf{3}$ & 4133 & 5926 & 51620 & 3846 & 4039 & 3664 \\
\hline $\mathbf{4}$ & 10331 & 10329 & -352 & 15519 & 15185 & 13934 \\
\hline $\mathbf{5}$ & 4133 & 10535 & 18618 & 3083 & 4235 & 3602 \\
\hline $\mathbf{6}$ & 413 & 1061 & 1927 & 313 & 430 & 365 \\
\hline $\mathbf{7}$ & 6612 & 16778 & 29270 & 4895 & 6723 & 5723 \\
\hline $\mathbf{8}$ & 1653 & -2963 & -6684 & 1950 & 1285 & 1721 \\
\hline $\mathbf{9}$ & 1240 & -3087 & -16478 & 1867 & 1076 & 1813 \\
\hline $\mathbf{1 0}$ & 1653 & -388 & -3053 & 2416 & 1804 & 2502 \\
\hline $\mathbf{1 1}$ & 4133 & -18958 & -43603 & 4609 & 589 & 3844 \\
\hline $\mathbf{1 2}$ & 827 & 1561 & -1954 & 668 & 824 & 746 \\
\hline $\mathbf{1 3}$ & 4133 & 13723 & 41679 & 5229 & 6546 & 5567 \\
\hline $\mathbf{1 4}$ & 620 & 2573 & 5652 & 838 & 1076 & 939 \\
\hline $\mathbf{1 5}$ & 4133 & 8668 & -11341 & 3971 & 4997 & 4440 \\
\hline $\mathbf{1 6}$ & 2066 & -9 & 5 & 3 & 1 & 2 \\
\hline $\mathbf{1 7}$ & 8265 & 14433 & 24278 & 5163 & 5975 & 5514 \\
\hline $\mathbf{1 8}$ & 4133 & 1412 & -23819 & 5385 & 5449 & 5479 \\
\hline $\mathbf{1 9}$ & 620 & 1616 & 3541 & 836 & 1009 & 908 \\
\hline $\mathbf{2 0}$ & 413 & -724 & -1230 & 259 & 140 & 213 \\
\hline CT & 65707 & 65707 & 65707 & 65707 & 65707 & 65707 \\
\hline
\end{tabular}

